\title{
Synthesis and Antioxidant Activity of 3-Methoxyflavones
}

\author{
Ho Yoo, ${ }^{\dagger}$ Seung Hwan Kim, ${ }^{\dagger}, \star$ Jiyong Lee, ${ }^{\dagger}$ Hyoung Ja Kim, ${ }^{\dagger}$ Seon Hee Seo, ${ }^{\dagger}$ \\ Bong Young Chung, ${ }^{\star}$ Changbae Jin, ${ }^{\dagger}$ and Yong Sup Lee ${ }^{\S, *}$ \\ ${ }^{\dagger}$ Life Sciences Division, Korea Institute of Science \& Technology, P.O. Box 131, Seoul 130-650, Korea \\ Department of Chemistry, Korea University, Seoul 136-701, Korea \\ ${ }^{\S}$ Kyung Hee East-West Pharmaceutical Research Institute and Department of Pharmaceutical Science, College of Pharmacy, \\ Kyung Hee University, Seoul 130-701, Korea. *E-mail: kyslee@khu.ac.kr \\ Received March 29, 2005
}

Key Words : Antioxidant, 3-O-Methoxyflavone, DPPH free radical, Superoxide anion radical, Lipid peroxidation

It is becoming increasingly apparent that the overproduction of reactive oxygen species may overwhelm the protective antioxidative defense mechanisms resulting in oxidative tissue injury. ${ }^{1}$ Reactive oxygen species have been implicated in several different diseases including ischemia, inflammation and cancer. ${ }^{2}$ Fortunately, plants contain a wide variety of free radical scavenging molecules such as flavonoids, anthocyanins, carotenoids, dietary glutathione, vitamins and endogenous metabolites. Such natural products are rich in antioxidant activities. ${ }^{3}$ Recently, important biological property of natural flavonoids was suggested mainly due to their antioxidant activity elicited by scavenging oxygen radicals and inhibiting peroxidation. ${ }^{4}$ Also, the antioxidant activity of the flavonoids varies considerably depending on the backbone structures and functional groups. $^{5}$

In the course of searching for neuroprotective agents, we recently identified quercetin 3-O-methyl ether $\left(\mathbf{1} \mathbf{b}, \mathrm{R}_{1}, \mathrm{R}_{3}\right.$, $\mathrm{R}_{4}=\mathrm{H}, \mathrm{R}_{2}=\mathrm{OH}$ ) as a potent antioxidant from Opuntia ficus-indica var. saboten. ${ }^{6}$ Quercetin 3-O-methyl ether also exhibited potent neuroprotective effects on the oxidative injuries to neuronal cells. ${ }^{7}$ For the purpose of the development of neuroprotective agents for therapeutic use, we needed to modify the structure or substitute suitable groups in the structure of $\mathbf{1 b}$ to improve physicochemical properties or enhance antioxidant activities. Therefore, we synthesized a series of 3-methoxyflavones (1c-g) and examined their antioxidant activities to elucidate a suitable position for modification (Figure 1). To investigate briefly the influence

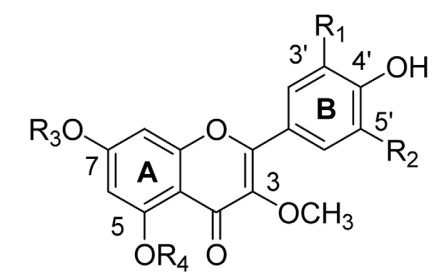

3-Methoxyflavones $(\mathbf{1} \mathbf{a} \sim \mathbf{f})$

$\mathrm{R}_{1}, \mathrm{R}_{2}=\mathrm{H}$ or $\mathrm{OH} ; \mathrm{R}_{3}, \mathrm{R}_{4}=\mathrm{H}$ or $\mathrm{CH}_{3}$

Figure 1 of substituents of 3-methoxyflavones on antioxidant activity, we methylated the C-5 or C-7 hydroxyl group at A-ring of the chromone backbone or introduced mono-, di-, and trihydroxyl groups at B-ring.

\section{Results and Discussion}

Chemistry. Kaempferol 3-O-methyl ether (1a) and quercetin 3-O-methyl ether (1b) were obtained from the ethyl acetate fraction of the stems of Opuntia ficus-indica var. saboten as previously reported by us. ${ }^{6}$ The other 3-O-methyl ether derivatives (1c-1f) were synthesized as illustrated in Scheme 1. $O$-Benzyl-protected flavone $4 \mathbf{c}$ was prepared in $76 \%$ yield by coupling acetophenone 2 with $O$-benzylprotected benzoyl chloride $\mathbf{3 c}$ followed by in situ cyclization of the resulting ester to form chromone ring in the presence of tetrabutylammonium hydrogen sulfate (TBAHS) under a basic condition. ${ }^{8}$ Deprotection of benzyl group in $\mathbf{4 c}$ using $\mathrm{Pd}(\mathrm{OH})_{2} / \mathrm{C}$ and cyclohexene afforded myricetin 3- $O$-methyl ether (1c) in $66 \%$ yield.

Selective mono-methylation at the C-7 position of benzylprotected 3-methoxyflavones $\mathbf{4 b}$ and $\mathbf{4 c}$ were performed using dimethyl sulfate and potassium carbonate in acetone at room temperature to provide $\mathbf{5 d}$ and $\mathbf{5 e}$ in $94 \%$ and $79 \%$ yields, respectively. ${ }^{9}$ Di-methylation of $\mathbf{4 b}$ was also achieved at reflux temperature to obtain $\mathbf{5 f}$ in $91 \%$ yield. The benzyl groups in 5d-f were removed using again $\mathrm{Pd}(\mathrm{OH})_{2}$ and cyclohexene to yield 1d-f in $72-87 \%$ yields.

To further investigate the influence of the $\mathrm{C}-7$ position on the antioxidant activity, we synthesized compound $\mathbf{1 g}$, which has no $\mathrm{C}-7$ hydroxyl group in quercetin 3-O-methyl ether as shown in Scheme 2. The C-7 hydroxyl group of $\mathbf{4 b}$ was selectively converted into the tetrazolyl ether 6 with 5chloro-1-phenyltetrazole, which was then reduced with formic acid and palladium on charcoal to lead $\mathbf{1 g .}{ }^{10}$

Biological Activity. Table 1 summarizes the results of the antioxidant activities obtained using three different bioassay systems; 1,1-diphenyl-2-picrylhydrazyl (DPPH) radical scavenging, ${ }^{11}$ superoxide anion radical scavenging ${ }^{12}$ and lipid peroxidation inhibition activity assays. ${ }^{13}$ Vitamin $\mathrm{C}$ and trolox were used for comparisons of activities in our assay 
<smiles>COCC(=O)c1c(O)cc(O)cc1O</smiles>

2

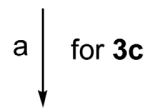<smiles>[R]c1cc(-c2oc3cc(O)cc(O)c3c(=O)c2OC)cc([R])c1OC</smiles>

4b, $R_{1}=H, R_{2}=H$

4c, $R_{1}=R_{2}=O B n$

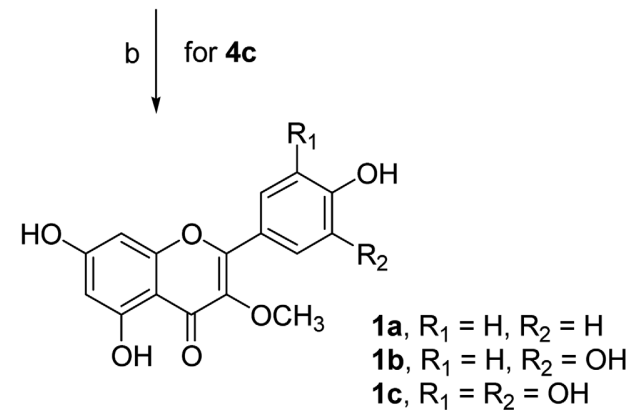

3a, $\mathrm{R}_{1}=\mathrm{H}, \mathrm{R}_{2}=\mathrm{H}$

3b, $R_{1}=H, R_{2}=O B n$

3c, $R_{1}=R_{2}=O B n$ dimethyl sulfate, acetone.<smiles>COc1cc(-c2oc3cc(Oc4nnnn4-c4ccccc4)cc(O)c3c(=O)c2OC)ccc1OCc1ccccc1</smiles>

for $4 b$ and $4 c$

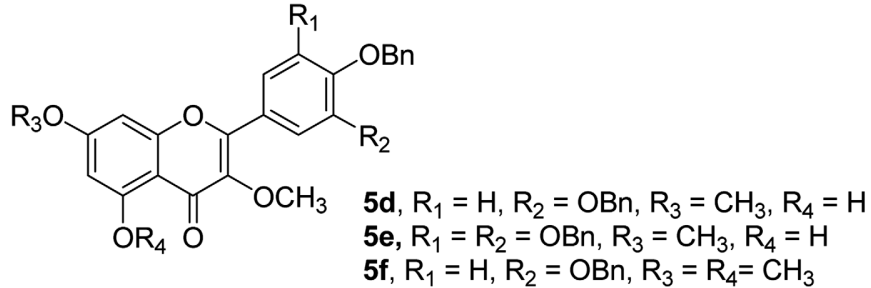<smiles>C1CCC1</smiles><smiles>[R]C=CC(=CC)c1oc2cc(O[R6])cc(O[R20])c2c(=O)c1O</smiles>

1d, $\mathrm{R}_{1}=\mathrm{H}, \mathrm{R}_{2}=\mathrm{OH}, \mathrm{R}_{3}=\mathrm{CH}_{3}, \mathrm{R}_{4}=\mathrm{H}$ 1e, $R_{1}=R_{2}=\mathrm{OH}, \mathrm{R}_{3}=\mathrm{CH}_{3}, \mathrm{R}_{4}=\mathrm{H}$ 1f, $R_{1}=H, R_{2}=O H, R_{3}=R_{4}=C_{3}$

Scheme 1. Reagents and conditions: (a) i. $\mathrm{K}_{2} \mathrm{CO}_{3}$, TBAHS, toluene, ii. reflux; (b) $\mathrm{Pd}(\mathrm{OH})_{2} / \mathrm{C}, \mathrm{EtOH} / \mathrm{cyclohexene:} 1 / 1$, reflux; (c) $\mathrm{K}_{2} \mathrm{CO}_{3}$,

$1 \mathrm{~g}$

Scheme 2. Reagents and conditions: (a) potassium tert-butoxide, 5-chloro-1-phenyltetrazole; (b) $\mathrm{HCOOH}, \mathrm{Pd} / \mathrm{C}$, benzene, EtOH, $\mathrm{H}_{2} \mathrm{O}$.

systems as hydrophilic and lipophilic antioxidants, respectively.

Almost 3-methoxyflavones except for 1a exhibited potent antioxidant activities in three different assay systems. The DPPH radical scavenging activities of synthesized compounds (1c-g) were comparable to the activity of parent compound, quercetin 3-O-methyl ether (1b) and vitamin $\mathrm{C}$. On the other hand, lipid peroxidation inhibition activities of the compounds were much more potent than that of trolox. The antioxidant activity data of 1a-c indicate that at least two hydroxyl groups are required in the B-ring of flavones for antioxidant activities. On methylation of the C-5 and/or C-7 hydroxyl groups in the A-ring (1d-f), the antioxidant activities were not much affected. However, when the C-7 hydroxyl group was removed from $\mathbf{1 b}$ as in $\mathbf{1 g}$, superoxide anion radical scavenging activity was reduced about 5-fold, while DPPH radical scavenging and lipid peroxidation inhibition activities were retained.

In conclusion, a series of 3-methoxyflavones (1a-g) were prepared and evaluated for the antioxidant activities. On methylation of the C-5 and/or C-7 hydroxyl groups in 3methoxyflavones, the antioxidant activities were retained, while removal of the C-7 hydroxyl group diminished superoxide anion radical scavenging activity. Therefore, the substitution of the functional group at the C-5 or C-7 position seems desired in the design of new antioxidative $3-$ methoxyflavones with improved physicochemical properties.

\section{Experimental Section}

Chemistry.

General: ${ }^{1} \mathrm{H}$ and ${ }^{13} \mathrm{C}$ NMR spectra were recorded on a 
Table 1. Antioxidant activities of 3-methoxyflavones (1a-g)

\begin{tabular}{|c|c|c|c|c|c|c|c|c|c|}
\hline \multirow[b]{2}{*}{ Entry } & \multirow[b]{2}{*}{ Structure } & \multicolumn{3}{|c|}{$\mathrm{IC}_{50}(\mu \mathrm{g} / \mathrm{mL})^{a}$} & \multirow[b]{2}{*}{ Entry } & \multirow[b]{2}{*}{ Structure } & \multicolumn{3}{|c|}{$\mathrm{IC}_{50}(\mu \mathrm{g} / \mathrm{mL})^{a}$} \\
\hline & & $\mathrm{DPPH}^{b}$ & $\begin{array}{l}\text { Superoxide } \\
\text { anion }^{c}\end{array}$ & $\begin{array}{c}\text { Lipid } \\
\text { peroxidation }^{d}\end{array}$ & & & $\mathrm{DPPH}^{b}$ & $\begin{array}{l}\text { Superoxide } \\
\text { anion }^{c}\end{array}$ & $\begin{array}{c}\text { Lipid } \\
\text { peroxidation }^{d}\end{array}$ \\
\hline 1a & & $>50$ & $>50$ & $>50$ & 1e & & $\begin{array}{c}5.48 \\
\pm 0.63\end{array}$ & $\begin{array}{c}7.10 \\
\pm 1.74\end{array}$ & $\begin{array}{c}3.78 \\
\pm 0.74\end{array}$ \\
\hline $1 b$ & & $\begin{array}{c}3.76 \\
\pm 0.22\end{array}$ & $\begin{array}{c}4.77 \\
\pm 0.14\end{array}$ & $\begin{array}{c}4.41 \\
\pm 1.06\end{array}$ & 1f & & $\begin{array}{c}4.20 \\
\pm 0.49\end{array}$ & $\begin{array}{c}4.78 \\
\pm 0.48\end{array}$ & $\begin{array}{c}6.84 \\
\pm 0.75\end{array}$ \\
\hline 1c & & $\begin{array}{c}4.91 \\
\pm 0.48\end{array}$ & $\begin{array}{c}5.07 \\
\pm 0.68\end{array}$ & $\begin{array}{c}4.33 \\
\pm 0.14\end{array}$ & $1 \mathrm{~g}$ & & $\begin{array}{c}4.99 \\
\pm 0.87\end{array}$ & $\begin{array}{r}24.17 \\
\pm 1.70\end{array}$ & $\begin{array}{c}8.27 \\
\pm 0.46\end{array}$ \\
\hline 1d & & $\begin{array}{c}4.12 \\
\pm 0.51\end{array}$ & $\begin{array}{c}6.22 \\
\pm 0.83\end{array}$ & $\begin{array}{c}4.84 \\
\pm 0.31\end{array}$ & Vitamin & & $\begin{array}{c}5.07 \\
\pm 0.73\end{array}$ & $>50$ & $>50$ \\
\hline & & & & & Trolox & & $\mathrm{NT}^{e}$ & $>50$ & $\begin{array}{c}26.4 \\
\pm 1.26\end{array}$ \\
\hline
\end{tabular}

$\overline{{ }^{a} \mathrm{IC}_{50} \text { values with standard deviation are at least from three independent experiments. }{ }^{b} \mathrm{DPPH} \text { radical scavenging activity. }{ }^{c} \text { Superoxide anion radical }}$ scavenging activity generated in the xanthine/xanthine oxidase system. ${ }^{d}$ Iron-dependent lipid peroxidation inhibition activity using rat liver homogenate. ${ }^{e}$ Not tested.

Gemini Varian-300 (300 and $75 \mathrm{MHz}$, respectively). Analytical thin layer chromatographies (TLC) were carried out by precoated silica gel (E. Merck Kiesegel $60 \mathrm{~F}_{254}$ layer thickness $0.25 \mathrm{~mm}$ ). Flash column chromatographies were performed with Merck Kiesegel 60 Art 9385 (230-400 mesh). All solvents used were purified according to standard procedures. Compounds $\mathbf{1 a}$ and $\mathbf{1 b}$ were obtained from the ethyl acetate fraction of the stems of Opuntia ficus-indica var. saboten as previously reported. ${ }^{6}$ Compounds $\mathbf{4 b}$ was prepared by the known procedure. ${ }^{7}$

3',4',5'-Tribenzyloxy-5,7-dihydroxy-3-methoxyflavone (4c). To a solution of 2',4',6'-trihydroxy-2-methoxyacetophenone (2, $1.0 \mathrm{~g}, 5.4 \mathrm{mmol})$, TBAHS (1.8 g, $5.4 \mathrm{mmol})$ and $\mathrm{K}_{2} \mathrm{CO}_{3}(1.4 \mathrm{~g}, 10.8 \mathrm{mmol})$ in toluene $(70 \mathrm{~mL})$ was added 3,4,5-tribenzyloxybenzoyl chloride (3c, $5.0 \mathrm{~g}, 11.0 \mathrm{mmol}$ ) portionwise at $0{ }^{\circ} \mathrm{C}$ and the solution was heated at $90{ }^{\circ} \mathrm{C}$ for $12 \mathrm{~h}$. The mixture was cooled and diluted with $\mathrm{H}_{2} \mathrm{O}$ and then extracted with $\mathrm{CH}_{2} \mathrm{Cl}_{2}$. The organic layer was washed with brine, dried over $\mathrm{MgSO}_{4}$, and concentrated. The residue was purified by flash column chromatography $\left(\mathrm{CH}_{2} \mathrm{Cl}_{2} / \mathrm{MeOH}=\right.$ 98 : 2) to afford $4 \mathrm{c}(2.4 \mathrm{~g}, 76 \%)$ as a solid. ${ }^{1} \mathrm{H}$ NMR $\left(\mathrm{DMSO}-d_{6}\right) \delta 12.54(1 \mathrm{H}$, br s, $O H), 7.49-7.26(17 \mathrm{H}, \mathrm{m}, H 2$ ', $\left.H_{6}^{\prime}, \mathrm{OCH}_{2} \mathrm{Ph}\right), 6.49(1 \mathrm{H}, \mathrm{s}, H 8), 6.22(1 \mathrm{H}, \mathrm{s}, H 6), 5.23(4 \mathrm{H}$, s, $\left.\mathrm{CH}_{2} \mathrm{Ph}\right), 5.08\left(2 \mathrm{H}, \mathrm{s},-\mathrm{CH}_{2} \mathrm{Ph}\right), 3.66\left(3 \mathrm{H}, \mathrm{s}, \mathrm{OCH}_{3}\right)$.

3',4'-Dibenzyloxy-3,7-dimethoxy-5-hydroxyflavone (5d). To a solution of compound $\mathbf{4 b}(0.2 \mathrm{~g}, 0.4 \mathrm{mmol})$ in acetone $(30 \mathrm{~mL})$ was added $\mathrm{K}_{2} \mathrm{CO}_{3}(2.0 \mathrm{~g}, 14.5 \mathrm{mmol})$ and dimethyl sulfate $(40 \mathrm{~mL}, 0.42 \mathrm{mmol})$. The reaction mixture was stirred for $24 \mathrm{~h}$ at room temperature and the mixture was filtered through Celite. The filtrate was concentrated, diluted with water and extracted with EtOAc. The organic layer was dried over $\mathrm{MgSO}_{4}$, concentrated, and purified by flash column chromatography $(\mathrm{EtOAc} / n$-hexane $=1: 5)$ to afford 5d $(0.19 \mathrm{~g}, 94 \%)$ as a solid. ${ }^{1} \mathrm{H}$ NMR $\left(\mathrm{CDCl}_{3}\right) \delta 12.61(1 \mathrm{H}$, br s, $O H), 7.75\left(1 \mathrm{H}, \mathrm{d}, J=2.0 \mathrm{~Hz}, H 2^{\prime}\right), 7.66(1 \mathrm{H}, \mathrm{dd}, J=$ 2.0, 8.6 Hz, H6'), 7.49-7.33 (10H, m, $\left.\mathrm{OCH}_{2} P h\right), 7.02(1 \mathrm{H}, \mathrm{d}$, $\left.J=8.6 \mathrm{~Hz}, H 5^{\prime}\right), 6.37(1 \mathrm{H}, \mathrm{d}, J=2.1 \mathrm{~Hz}, H 8), 6.33(1 \mathrm{H}, \mathrm{d}, J$ $=2.1 \mathrm{~Hz}, H 6), 5.26\left(2 \mathrm{H}, \mathrm{s}, \mathrm{OCH}_{2} \mathrm{Ph}\right), 5.24\left(2 \mathrm{H}, \mathrm{s}, \mathrm{OCH}_{2} \mathrm{Ph}\right)$, $3.86\left(3 \mathrm{H}, \mathrm{s}, \mathrm{OCH}_{3}\right), 3.70\left(3 \mathrm{H}, \mathrm{s}, \mathrm{OCH}_{3}\right)$.

3',4',5'-Tribenzyloxy-3,7-dimethoxy-5-hydroxyflavone (5e). To a solution of compound $4 \mathbf{c}(0.2 \mathrm{~g}, 0.33 \mathrm{mmol})$ in acetone $(30 \mathrm{~mL})$ was added $\mathrm{K}_{2} \mathrm{CO}_{3}(2.0 \mathrm{~g}, 14.5 \mathrm{mmol})$ and dimethyl sulfate $(31 \mathrm{~mL}, 0.33 \mathrm{mmol})$. The reaction mixture was stirred for $24 \mathrm{~h}$ at room temperature and the mixture was filtered through Celite. The filtrate was concentrated, diluted with water, and extracted with EtOAc. The organic layer was dried over $\mathrm{MgSO}_{4}$, concentrated, and purified by flash column chromatography $(\mathrm{EtOAc} / n$-hexane $=1: 5)$ to afford 5e $(0.16 \mathrm{~g}, 79 \%)$ as a solid. ${ }^{1} \mathrm{H} \mathrm{NMR}\left(\mathrm{CDCl}_{3}\right) \delta 12.55(1 \mathrm{H}, \mathrm{s}$, $\mathrm{OH}), 7.46-7.25$ (17H, m, H2', H6', $\left.\mathrm{OCH}_{2} \mathrm{Ph}\right), 6.36(1 \mathrm{H}, \mathrm{d}, J$ $=2.2 \mathrm{~Hz}, H 8), 6.35(1 \mathrm{H}, \mathrm{d}, J=2.2 \mathrm{~Hz}, H 6), 5.19(6 \mathrm{H}, \mathrm{s}$, $\left.\mathrm{OCH}_{2} \mathrm{Ph}\right), 3.88\left(3 \mathrm{H}, \mathrm{s}, \mathrm{OCH}_{3}\right), 3.66\left(3 \mathrm{H}, \mathrm{s}, \mathrm{OCH}_{3}\right)$.

3',4'-Dibenzyloxy-3,5,7-trimethoxyflavone (5f). To a solution of compound $\mathbf{4 b}(0.2 \mathrm{~g}, 0.4 \mathrm{mmol})$ in acetone (30 $\mathrm{mL})$ was added $\mathrm{K}_{2} \mathrm{CO}_{3}(3.0 \mathrm{~g}, 21.7 \mathrm{mmol})$ and dimethyl sulfate $(71 \mathrm{~mL}, 0.74 \mathrm{mmol})$. The reaction mixture was refluxed for $2 \mathrm{~h}$ and the mixture was filtered through Celite. The filtrate was concentrated, diluted with water and 
extracted with EtOAc. The organic layer was dried over $\mathrm{MgSO}_{4}$, concentrated, and purified by flash column chromatography $($ EtOAc $/ n$-hexane $=3: 1)$ to afford $3 \mathbf{e}(0.19 \mathrm{~g}, 91 \%)$ as a solid. ${ }^{1} \mathrm{H} \mathrm{NMR}\left(\mathrm{CDCl}_{3}\right) \delta 7.79\left(1 \mathrm{H}, \mathrm{d}, J=2.1 \mathrm{~Hz}, H 2^{\prime}\right)$, $7.66\left(1 \mathrm{H}, \mathrm{dd}, J=2.1,8.6 \mathrm{~Hz}, H 6^{\prime}\right), 7.52-7.34(10 \mathrm{H}, \mathrm{m}$, $\left.\mathrm{OCH}_{2} P h\right), 7.02\left(1 \mathrm{H}, \mathrm{d}, J=8.6 \mathrm{~Hz}, H 5^{\prime}\right), 6.42(1 \mathrm{H}, \mathrm{d}, J=2.1$ $\mathrm{Hz}, H 8), 6.31(1 \mathrm{H}, \mathrm{d}, J=2.1 \mathrm{~Hz}, H 6), 5.26\left(4 \mathrm{H}, \mathrm{s}, \mathrm{OCH}_{2} \mathrm{Ph}\right)$, $3.94\left(3 \mathrm{H}, \mathrm{s}, \mathrm{OCH}_{3}\right), 3.88\left(3 \mathrm{H}, \mathrm{s}, \mathrm{OCH}_{3}\right), 3.76\left(3 \mathrm{H}, \mathrm{s}, \mathrm{OCH}_{3}\right)$.

3',4',5-Trihydroxy-3-methoxyflavone (1c). To a solution of $4 \mathbf{c}(150 \mathrm{mg}, 0.25 \mathrm{mmol})$ in mixture of solvents (ethanol/ cyclohexene $=5: 1,6 \mathrm{~mL})$ was added excess $\mathrm{Pd}(\mathrm{OH})_{2} / \mathrm{C}(25$ $\mathrm{mg}$ ) and heated at $60-70{ }^{\circ} \mathrm{C}$ for $1 \mathrm{~h}$. The mixture was filtered through Celite and concentrated. The residue was purified by flash column chromatography $\left(\mathrm{CH}_{2} \mathrm{Cl}_{2} / \mathrm{MeOH}=15: 1\right)$ to afford $1 \mathrm{c}(55 \mathrm{mg}, 66 \%)$ as a solid. ${ }^{1} \mathrm{H}$ NMR (DMSO- $\left.d_{6}\right) \delta$ $12.82(1 \mathrm{H}$, br s, $O H), 7.23$ (2H, s, $\left.H 2^{\prime}, H 6^{\prime}\right), 6.48$ (1H, d, $J=$ $1.9 \mathrm{~Hz}, H 8), 6.29(1 \mathrm{H}, \mathrm{d}, J=1.9 \mathrm{~Hz}, H 6), 3.79(3 \mathrm{H}, \mathrm{s}$, $\mathrm{OCH}_{3}$ ).

3',4',5-Trihydroxy-3,7-dimethoxyflavone (1d). By using the similar procedure for 1c, compound 1d was obtained from $5 \mathbf{d}(120 \mathrm{mg}, 0.24 \mathrm{mmol})$ and $\mathrm{Pd}(\mathrm{OH})_{2} / \mathrm{C}(24 \mathrm{mg})$ as a solid in $97 \%$ yield $(75 \mathrm{mg}) .{ }^{1} \mathrm{H}$ NMR (DMSO- $\left.d_{6}\right) \delta 7.65$ $\left(1 \mathrm{H}, \mathrm{d}, J=1.4 \mathrm{~Hz}, H 2^{\prime}\right), 7.55\left(1 \mathrm{H}, \mathrm{dd}, J=1.4,8.4 \mathrm{~Hz}, H 6^{\prime}\right)$, $6.97\left(1 \mathrm{H}, \mathrm{d}, J=8.4 \mathrm{~Hz}, H 5^{\prime}\right), 6.77(1 \mathrm{H}, \mathrm{d}, J=1.9 \mathrm{~Hz}, H 8)$, $6.43(1 \mathrm{H}, \mathrm{d}, J=1.9 \mathrm{~Hz}, H 6), 3.93\left(3 \mathrm{H}, \mathrm{s}, \mathrm{OCH}_{3}\right), 3.86(3 \mathrm{H}$, $\left.\mathrm{s}, \mathrm{OCH}_{3}\right)$.

3',4',5',5-Tetrahydroxy-3,7-dimethoxyflavone (1e). Вy using the similar procedure for 1c, compound 1e was obtained from 5 e $(112 \mathrm{mg}, 0.18 \mathrm{mmol})$ and $\mathrm{Pd}(\mathrm{OH})_{2} / \mathrm{C}(12$ $\mathrm{mg}$ ) as a solid in $72 \%$ yield $(45 \mathrm{mg}) .{ }^{1} \mathrm{H}$ NMR (DMSO- $\left.d_{6}\right) \delta$ $12.70(1 \mathrm{H}$, br s, $O H), 7.16\left(2 \mathrm{H}, \mathrm{s}, H 2^{\prime}, H 6^{\prime}\right), 6.65(1 \mathrm{H}$, br s, H8), $6.36\left(1 \mathrm{H}\right.$, br s, H6), $3.85\left(3 \mathrm{H}, \mathrm{s}, \mathrm{OCH}_{3}\right), 3.78(3 \mathrm{H}, \mathrm{s}$, $\left.\mathrm{OCH}_{3}\right)$.

3',4'-Dihydroxy-3,5,7-trimethoxyflavone (1f). By using the similar procedure for $\mathbf{1 c}$, compound $\mathbf{1 f}$ was obtained from $5 \mathbf{5 f}(125 \mathrm{mg}, 0.24 \mathrm{mmol})$ and $\mathrm{Pd}(\mathrm{OH})_{2} / \mathrm{C}(26 \mathrm{mg})$ as a solid in $87 \%$ yield $(71 \mathrm{mg}) .{ }^{1} \mathrm{H}$ NMR (DMSO- $\left.d_{6}\right) \delta 7.53$ (1H, br s, H2'), $7.41\left(1 \mathrm{H}, \mathrm{d}, J=7.4 \mathrm{~Hz}, H 6^{\prime}\right), 6.87(1 \mathrm{H}, \mathrm{d}, J=$ $\left.7.4 \mathrm{~Hz}, H 5^{\prime}\right), 6.72(1 \mathrm{H}, \mathrm{s}, H 8), 6.45(1 \mathrm{H}, \mathrm{s}, H 6), 3.86(3 \mathrm{H}, \mathrm{s}$, $\left.\mathrm{OCH}_{3}\right), 3.82\left(3 \mathrm{H}, \mathrm{s}, \mathrm{OCH}_{3}\right), 3.70\left(3 \mathrm{H}, \mathrm{s}, \mathrm{OCH}_{3}\right)$.

3',4'-Dibenzyloxy-3-methoxy-7-[(1-phenyl-tetrazol-5yl)oxy]flavone (6). To a solution of $\mathbf{4 b}(100 \mathrm{mg}, 0.20 \mathrm{mmol})$ in dry DMF (1.5 mL) was added potassium tert-butoxide (41 $\mathrm{mg}, 0.35 \mathrm{mmol})$ at room temperature. When the base was dissolved, 5-chloro-1-phenyltetrazole ( $58 \mathrm{mg}, 0.35 \mathrm{mmol}$ ) in dry DMF $(1 \mathrm{~mL})$ was added to the solution. The mixture was stirred at room temperature for $10 \mathrm{~h}$ and poured into ice water. The mixture was extracted with EtOAc and the organic layer was washed with brine, dried over $\mathrm{MgSO}_{4}$, and concentrated. The residue was purified by flash column chromatography $(\mathrm{EtOAc} / n$-hexane $=1: 2)$ to afford $6(72$ $\mathrm{mg}, 56 \%)$ as a solid. ${ }^{1} \mathrm{H} \mathrm{NMR}\left(\mathrm{CDCl}_{3}\right) \delta 7.82-7.36(7 \mathrm{H}, \mathrm{m}$, tetrazole- $\left.P h, H 2^{\prime}, H 6^{\prime}\right), 7.27(1 \mathrm{H}, \mathrm{d}, J=1.5 \mathrm{~Hz}, H 8), 7.08$ $\left(1 \mathrm{H}, \mathrm{d}, J=8.7 \mathrm{~Hz}, H 5^{\prime}\right), 6.81(1 \mathrm{H}, \mathrm{d}, J=1.5 \mathrm{~Hz}, H 6), 5.32$ $\left(2 \mathrm{H}, \mathrm{s}, \mathrm{OCH}_{2} \mathrm{Ph}\right), 5.31\left(2 \mathrm{H}, \mathrm{s}, \mathrm{OCH}_{2} \mathrm{Ph}\right), 3.77(3 \mathrm{H}, \mathrm{s}$, $\left.\mathrm{OCH}_{3}\right)$.

3',4',5-Trihydroxy-3-methoxyflavone (1g). To a vigorously stirred solution of $6(66 \mathrm{mg}, 0.10 \mathrm{mmol})$ in benzene $(2.3 \mathrm{~mL}), \mathrm{H}_{2} \mathrm{O}(2.5 \mathrm{~mL})$, and $\mathrm{EtOH}(4.6 \mathrm{~mL})$ was added $\mathrm{Pd} /$ $\mathrm{C}(10 \%, 80 \mathrm{mg})$. HCOOH $(2 \mathrm{~mL})$ was then added and the mixture was refluxed at $100{ }^{\circ} \mathrm{C}$ for $3 \mathrm{~h}$. The solution was cooled, filtered through Celite, and concentrated. The residue was purified by flash column chromatography $\left(\mathrm{CH}_{2} \mathrm{Cl}_{2} / \mathrm{MeOH}=15: 1\right)$ to afford $1 \mathrm{~g}(8 \mathrm{mg}, 26 \%)$ as a solid. ${ }^{1} \mathrm{H} \mathrm{NMR}\left(\mathrm{CD}_{3} \mathrm{OD}\right) \delta 7.70\left(1 \mathrm{H}, \mathrm{d}, J=2.4 \mathrm{~Hz}, H 2{ }^{\prime}\right), 7.63(1 \mathrm{H}$, dd, $\left.J=2.4,8.1 \mathrm{~Hz}, H 6^{\prime}\right), 7.60(1 \mathrm{H}, \mathrm{t}, J=8.1 \mathrm{~Hz}, H 7), 7.06$ (1H, dd, $J=1.2,8.4 \mathrm{~Hz}, H 8), 6.94\left(1 \mathrm{H}, \mathrm{d}, J=8.1 \mathrm{~Hz}, H 5^{\prime}\right)$, $6.77(1 \mathrm{H}, \mathrm{dd}, J=1.2,8.4 \mathrm{~Hz}, \mathrm{H6}), 6.77\left(3 \mathrm{H}, \mathrm{s}, \mathrm{OCH}_{3}\right)$.

Acknowledgments. This research was supported by a grant (M1-0310-22-0001) from Bio-challenger Program funded by Ministry of Commerce, Industry and Energy of Korean government.

\section{References and Notes}

1. Haraguchi, H.; Ishikawa, H.; Sanchez, Y.; Ogura, T.; Kubo, Y.; Kubo, I. Bioorg. Med. Chem. 1997, 5, 865.

2. Cai, Q.; Rahn, R. O.; Zhang, R. Cancer Lett. 1997, 119, 99.

3. Choi, C. W.; Kim, S. C.; Hwang, S. S.; Choi, B. K.; Ahn, H. J.; Lee, M. Y.; Park, S. H.; Kim, S. K. Plant Science 2002, 163, 1161.

4. Braca, A.; Fico, G.; Morelli, I.; De Simone, F.; Tomè, F.; De Tommasi, N. J. Ethnopharm. 2003, 86, 63.

5. Dugas, Jr., A. J.; Castaneda-Acosta, J.; Bonin, G. C.; Price, K. L.; Fischer, N. H.; Winston, G. W. J. Nat. Prod. 2000, 63, 327.

6. Lee, E. H.; Kim, H. J.; Song, Y. S.; Jin, C.; Lee, K.-T.; Cho, J.; Lee, Y. S. Arch. Pharm. Res. 2003, 26, 1018.

7. Dok-Go, H.; Lee, K. H.; Kim, H. J.; Lee, E. H.; Lee, J.; Song, Y. S.; Lee, Y.-H.; Jin, C.; Lee, Y. S.; Cho, J. Brain Research 2003, 965(1-2), 130.

8. Boers, F.; Deng, B.-L.; Lemiere, G.; Lepoivre, J.; De Groot, A.; Dommisse, R.; Vlietinck, A. J. Arch. der Pharm. 1997, 330, 313.

9. Bouktaib, M.; Lebrun, S.; Atmani, A.; Rolando, C. Tetrahedron 2002, 58, 10001.

10. De Meyer, N.; Haemers, A.; Mishra, L.; Pandey, H. K.; Pieters, L. A. C.; Berghe, D. A. V.; Vlietinck, A. J. J. Med. Chem. 1991, 34, 736.

11. Lee, J. S.; Kim, H. J.; Park, H.; Lee, Y. S. J. Nat. Prod. 2002, 65, 1367.

12. Toda, S.; Kumura, M.; Ohnishi, M. Planta Med. 1991, 57, 8.

13. Sanz, M. J.; Ferrandiz, M. L.; Cejudo, M.; Terencio, M. C.; Gil, B.; Bustos, G.; Ubeda, A.; Gunasegaran, R.; Alcaraz, M. J. Xenobiotica 1994, 24, 689. 